\title{
Análise de Custo-efetividade e Impacto Orçamentário de Anticoagulantes no Tratamento da Trombose Venosa Profunda em Pacientes Oncológicos
}

doi: https://doi.org/10.32635/2176-9745.RBC.2019v65n3.295

\author{
Analysis of Cost-effectiveness and Budget Impact of Anticoagulants in the Treatment of Deep Venous Thrombosis in Oncology \\ Patients \\ Análisis de Costo-efectividad e Impacto Presupuestal de Anticoagulantes en el Tratamiento de la Trombosis Venosa \\ Profunda en Pacientes Oncológicos
}

\author{
Thamiris Brandão Peixoto Sampaio'; Marcos Jose Pereira Renni ${ }^{2}$; Rodrigo Saar da Costa ${ }^{3}$
}

\begin{abstract}
Resumo
Introdução: A trombose venosa profunda é uma complicação comum e intimamente relacionada às neoplasias. Novos anticoagulantes orais foram lançados nos últimos anos, entre eles, a rivaroxabana. Objetivo: $\mathrm{O}$ estudo analisou o custo-efetividade e o impacto orçamentário da rivaroxabana versus enoxaparina. Método: Trata-se de uma coorte retrospectiva, realizada com população oncológica sob a perspectiva do Sistema Único de Saúde. Por meio do modelo de árvore de decisão, foram comparados desfechos de sangramento e retrombose, e custos do tratamento da trombose venosa profunda com rivaroxabana ou enoxaparina, em um horizonte temporal de sete meses. Custos diretos foram extraídos do Sistema de Gerenciamento da Sistema de Gerenciamento da Tabela de Procedimentos, Medicamentos, Órteses, Próteses e Materiais e Medicamentos Especiais do SUS (SIGTAP-SUS), e empregou-se a Planilha Brasileira de Impacto Orçamentário de tecnologias da saúde para avaliação do impacto orçamentário com base na população brasileira de 2017, em cinco anos. A análise de sensibilidade simulou cenários tanto na avaliaçáo de custo-efetividade quanto na de impacto orçamentário. Resultados: Cento e cinquenta e três pacientes foram incluídos na análise de custo-efetividade com diversas neoplasias. A rivaroxabana demonstrou não inferioridade terapêutica comparada à enoxaparina. A razão de custo-efetividade incremental foi de $\mathrm{R} \$ 5.521,71$ por unidade de benefício ganho com a nova alternativa, rivaroxabana. $\mathrm{Na}$ análise de sensibilidade, a rivaroxabana manteve-se dominante. Foi demonstrada uma economia no impacto orçamentário incremental de R \$ 85.950.791.129,21 com a utilizaçáo de rivaroxabana ao longo de cinco anos em comparação ao cenário de referência, e esta se manteve como opção mais econômica perante as análises de sensibilidade. Conclusáo: A rivaroxabana, nesse contexto, apresentou-se como uma importante alternativa terapêutica.
\end{abstract}

Palavras-chave: Farmacoeconomia; Neoplasias; Trombose Venosa; Anticoagulantes; Sistema Único de Saúde.

\section{Abstract}

Introduction: Deep vein thrombosis is a common complication and closely related to neoplasms. New oral anticoagulants have been launched in recent years, among them rivaroxaban. Objective: The study analyzed the costeffectiveness and budget impact of rivaroxaban versus enoxaparin. Method: This is a retrospective cohort, performed with oncological population from the perspective of Sistema Único de Saúde (National Health System). The decision tree model compared outcomes of bleeding and rethrombosis, and costs of treatment of deep venous thrombosis with rivaroxaban or enoxaparin in a time horizon of seven months. Direct costs were extracted from the SIGTAP-SUS, and the Brazilian Spreadsheet for Budgetary Impact of Health Technologies was used to evaluate the budgetary impact based in the Brazilian population of 2017 over a five-year period. The sensitivity analysis simulated scenarios for both cost-effectiveness and budget impact assessments. Results: One hundred and fifty-three patients were included in the cost-effectiveness analysis with several neoplasms. Rivaroxaban demonstrated no therapeutic inferiority compared to enoxaparin. The incremental cost-effectiveness ratio was $\mathrm{R} \$ 5,521.71$ per benefit unit spared with the new alternative, rivaroxaban. In the sensitivity analysis, rivaroxaban remained dominant. An economy in incremental budget impact of $\mathrm{R} \$ 85,950,791,129.21$ was demonstrated with the use of rivaroxaban over five years in comparison to the reference scenario, and this continued as the most economic option in relation to sensitivity analyzes. Conclusion: In this context rivaroxaban was an important therapeutic alternative.

Key words: Economics, Pharmaceutical; Neoplasms; Venous Thrombosis; Anticoagulants; Unified Health System.
Resumen

Introducción: La trombosis venosa profunda es una complicación común e íntimamente relacionada a las neoplasias. Los nuevos anticoagulantes orales fueron lanzados en los últimos ańos, entre ellos la rivaroxabana. Objetivo: El estudio analizó el Costo-Efectividad y el Impacto Presupuestario de la rivaroxabana versus enoxaparina. Método: En el modelo de árbol de decisión se compararon los resultados de la hemorragia y la retrombosis, y los costos del tratamiento de la trombosis venosa profunda con rivaroxabana o enoxaparina, con una cohorte retrospectiva, realizada con población oncológica bajo la perspectiva del Sistema Único de Salud en un horizonte temporal de siete meses. Los costos directos fueron extraídos del SIGTAP-SUS, y se empleó la Planilla Brasileña de Impacto Presupuestario de Tecnologías de la Salud para evaluación del Impacto Presupuestario con base en la población brasileńa de 2017 en un horizonte temporal de cinco años. El análisis de sensibilidad simuló escenarios tanto en la evaluación de Costo-Efectividad y en la de Impacto Presupuestario. Resultados: Ciento cincuenta y tres pacientes fueron incluidos en el análisis de CostoEfectividad con diversas neoplasias. La rivaroxabana demostró no inferioridad terapéutica comparada a la enoxaparina. La razón de costo-efectividad incremental fue de R \$ 5.521,71 por unidad de beneficio ganada con la nueva alternativa, rivaroxabana. En el análisis de sensibilidad, la rivaroxabana se mantuvo dominante. Se demostró una economía em el Impacto Presupuestario incremental de R \$ 85.950.791.129,21 con la utilización de rivaroxabana a lo largo de 5 años en comparación al escenario de referencia, y ésta se mantuvo como opción más económica ante los análisis de sensibilidad. Conclusión: La rivaroxabana, en este contexto, se presentó como una importante alternativa terapéutica.

Palabras clave: Economía Farmacéutica; Neoplasias; Trombosis de la Vena; Anticoagulantes; Sistema Único de Salud.

${ }^{1}$ Instituto Nacional de Câncer José Alencar Gomes da Silva (INCA). Rio de Janeiro (RJ), Brasil. Orcid iD: https://orcid.org/0000-0001-8186-1184 ${ }^{2}$ INCA. Rio de Janeiro (RJ), Brasil. Orcid iD: https://orcid.org/0000-0003-3381-7394 ${ }^{3}$ INCA. Rio de Janeiro (RJ), Brasil. Orcid iD: https://orcid.org/0000-0002-1719-6949 Endereço para correspondência: Rodrigo Saar da Costa. Serviço de Farmácia do Hospital do Câncer l/INCA. Praça Cruz Vermelha, 23 - 70 andar - Centro. Rio de Janeiro (RJ), Brasil. CEP 20230-130. E-mail: saarrc@gmail.com 


\section{INTRODUÇÃO}

O tromboembolismo venoso (TEV) é uma patologia que compreende a trombose venosa profunda (TVP) e o tromboembolismo pulmonar (TEP). A relação entre câncer e estado de hipercoagulação sanguínea foi descrita inicialmente por Armand Trousseau em 1865, e tem sido amplamente discutida desde então. $\mathrm{O}$ risco de um paciente com câncer desenvolver TEV depende de inúmeras variáveis; entre elas, o tipo de tumor, estadiamento, momento da evolução clínica, tratamento realizado e fatores intrínsecos relacionados ao indivíduo ${ }^{1,2}$.

A TVP é uma complicação comum em pacientes com câncer e está associada a importantes taxas de morbidade e mortalidade, assim como a elevados custos de tratamento. Os eventos tromboembólicos em pacientes com câncer são em grande maioria manifestaçôes de trombose venosa, como a TVP, em membros inferiores e/ou embolia pulmonar ${ }^{3-5}$.

A trombose é a segunda causa de óbito na maioria dos tipos de neoplasias, e estudos epidemiológicos têm demonstrado uma significativa correlação entre a ocorrência de trombose e um pior prognóstico da doença ${ }^{6,7}$.

No que se refere ao tratamento farmacoterapêutico, diretrizes internacionais como as da American Society of Clinical Oncology (Asco), da European Society for Medical Oncology (Esmo) e do International Society on Thrombosis and Haemostasis (ISTH) ainda consideram a heparina de baixo peso molecular, a exemplo da enoxaparina, como opçóes farmacológicas de primeira linha para o tratamento da TVP em pacientes oncológicos ${ }^{8,9}$.

Todavia, considerando as dificuldades no manejo, custos, desconforto para os pacientes e o avanço tecnológico, novos anticoagulantes orais inibidores diretos dos fatores de coagulação foram lançados no mercado farmacêutico, a exemplo da rivaroxabana, inibidor direto altamente seletivo do fator $\mathrm{Xa}^{10}$. Nesse contexto, existem alguns estudos clínicos, como o Einstein DVT, que demonstram a não inferioridade da rivaroxabana diante da terapia-padrão enoxaparina no tratamento da TVP, e o Select- $D$, no qual a rivaroxabana apresentou semelhante ou melhor eficácia quando comparado à dalteparina em pacientes oncológicos com trombose ${ }^{11-14}$.

Entretanto, existem algumas contraindicações de uso da rivaroxabana; entre elas, podem ser citadas a utilização em pacientes com clearance inferior a $15 \mathrm{~mL} /$ minuto, doença hepática associada à coagulopatia, gestantes e em mulheres durante o período de amamentação.

No que diz a respeito à diretriz nacional, a I Diretriz Brasileira de Cardio-Oncologia da Sociedade Brasileira de Cardiologia, publicada no ano de 2013, não aborda a utilização de anticoagulantes orais de ação direta (DOAC) no tratamento de trombose associado a câncer. Em contrapartida, algumas diretrizes internacionais já sugerem a utilização desses novos fármacos; entre elas, as recomendaçốes apresentadas no ISTH de 2018, nas quais Khorana et al. ${ }^{15}$ recomendam o uso de DOAC como a rivaroxabana e edoxabana para o tratamento de TEV agudo em pacientes oncológicos em casos de baixo risco de sangramento e ausência de interaçóes medicamentosas. Essa recomendação se baseia no fato de que esses medicamentos possuem estudos robustos quanto ao tratamento de TVP, com eficácia e segurança comparáveis à terapia-padrão com heparina de baixo peso molecular, nessa população ${ }^{15}$.

Quanto ao aspecto econômico, há poucas publicaçôes com análises de custo-efetividade e impacto orçamentário que incluam pacientes oncológicos em uso de rivaroxabana sob a perspectiva do Sistema Único de Saúde (SUS). Em 2016, foi realizada uma análise de custo-efetividade sob a perspectiva de um hospital federal do SUS. Foram considerados os desfechos de efetividade e segurança da rivaroxabana e da enoxaparina em tratamento ambulatorial, sendo os dados clínicos extraídos de uma coorte retrospectiva de pacientes com câncer ginecológico e TVP. A rivaroxabana foi a tecnologia dominante, com uma economia de $\mathrm{R} \$ 7.789,61$ por paciente tratado ${ }^{16}$.

Do ponto de vista do Sistema Suplementar de Saúde, um estudo de custo-efetividade e impacto orçamentário produzido por da Silva ${ }^{17}$, comparando a rivaroxabana com dabigatrana e enoxaparina para a profilaxia de trombose secundária à cirurgia de artroplastia de quadril e joelho, observou que a rivaroxabana demonstrou ser a melhor opção para a profilaxia, em virtude da redução de custos. Outro estudo de impacto orçamentário realizado ${ }^{18}$ concluiu que a utilização da rivaroxabana no tratamento da TEV possui potencial econômico quando comparada à terapia com enoxaparina/varfarina também sob a visão do Sistema de Saúde Suplementar do Brasil e, além disso, constatou que o principal motivo de economia foi a redução no tempo de hospitalização.

Nesse contexto, a incorporação de um anticoagulante oral é concebida positivamente por facilitar a administração do medicamento, reduzir o número de consultas ambulatoriais, pela não necessidade de exames laboratoriais para ajuste de dose, por representar uma via não invasiva, apesar de possuir a contraindicação a pacientes com clearence inferior a $15 \mathrm{ml} / \mathrm{min}$ e em período de quimioterapia ${ }^{3}$.

Diante disso, o objetivo deste estudo foi realizar a avaliação de custo-efetividade da rivaroxabana versus terapia-padrão, enoxaparina, em uma populaçáo oncológica de maior abrangência, e uma análise de 
impacto orçamentário que visou a comparar os custos da utilização de ambas as tecnologias frente a diferentes cenários, a partir da perspectiva de um gestor de saúde, com o intuito de auxiliar na padronizaçáo de anticoagulantes em instituições hospitalares, levando-se em consideração não somente os custos, mas a segurança, a efetividade e o acesso a novas tecnologias.

\section{MÉTODO}

A estrutura metodológica empregada neste estudo foi dividida em duas etapas, análise de custo-efetividade e impacto orçamentário.

\section{ANÁLISE DE CUSTO-EFETIVIDADE}

$\mathrm{O}$ estudo compreende uma análise de uma coorte retrospectiva de um hospital federal especializado em oncologia, sob a perspectiva do gestor público. Foram incluídos no estudo pacientes oncológicos com TVP, idade superior a 18 anos e sob o tratamento anticoagulante com rivaroxabana ou enoxaparina pelo tempo mínimo de três meses consecutivos. Foram excluídos pacientes encaminhados para tratamento em outra instituição, pacientes com doses profiláticas de enoxaparina, anticoagulados por fibrilação atrial, acidente vascular cerebral e embolia pulmonar, bem como aqueles que não obedeceram aos critérios citados anteriormente. Dados clínicos como idade, tipo de tumor, estadiamento, tipo de tratamento recebido (cirurgia, radioterapia e quimioterapia), data do diagnóstico de trombose, tempo de duração da farmacoterapia anticoagulante, datas dos episódios de sangramento e TVP recorrente foram obtidos por meio de prontuários, no período de janeiro de 2017 a julho de 2017. Além disso, foram agregados a este estudo os dados primários da população oncológica analisada por Leira et al. ${ }^{16}$ com o intuito de ampliar a análise e garantir maior robustez aos dados.

O esquema de prescrições padronizado para rivaroxabana foi de $30 \mathrm{mg}$ diários $(15 \mathrm{mg}$ de $12 \mathrm{em} 12$ horas) durante os 21 dias, seguido de $20 \mathrm{mg}$ uma vez ao dia nos dias subsequentes, conforme recomendações em bula; e, para enoxaparina, $1 \mathrm{mg} / \mathrm{kg}$ a cada 12 horas ou $1,5 \mathrm{mg} / \mathrm{kg} / \mathrm{dia}^{19}$. Foram analisados desfechos de segurança e efetividade, respectivamente, sangramento clínico relevante e retrombose, baseados no estudo pivotal Einstein e Select- $D^{12,14}$. Para classificação dos episódios de sangramento, o ISTH foi utilizado ${ }^{20} \mathrm{e}$, para a classificação de retrombose, foi considerado o critério do algoritmo do Chest ${ }^{21}$.

A partir da coleta de dados, foi construída uma árvore de decisão (Figura 1) na qual foram aplicadas as probabilidades associadas aos eventos clínicos junto aos seus respectivos custos. Foi assumido um modelo de desfecho de sangramento seguido de TVP recorrente, e não foi considerada recorrência dos eventos em um horizonte temporal de sete meses de tratamento. Dessa forma, não houve necessidade de aplicação da taxa de desconto em razão do curto período de acompanhamento.

Os custos diretos foram obtidos a partir de valores de reembolso do SUS registrados no sítio do Sistema de Gerenciamento da Tabela de Procedimentos, Medicamentos, Órteses, Próteses e Materiais e Medicamentos Especiais do SUS (SIGTAP-SUS) e de atas de pregóes do Instituto Nacional de Câncer José Alencar Gomes da Silva (INCA), de janeiro a dezembro de 2017.

Para o cálculo de efetividade (E) das tecnologias analisadas, considerou-se o número de pacientes que apresentaram episódio de retrombose $(\mathrm{P}), \operatorname{logo} \mathrm{E}=(1-\mathrm{P})^{14}$.

Foram levadas com consideração uma média de exames doppler fluxometria e as consultas médicas no período de sete meses para cada paciente, em cada braço de tratamento, enoxaparina ou rivaroxabana. Para o cálculo de custo de tratamento de cada tecnologia, foram somados os valores da utilizaçáo de cada anticoagulante durante sete meses aos custos das médias de consultas e exames.

A fim de avaliar a robustez do modelo, foi realizada a análise de sensibilidade com diferentes cenários aplicados univariado e bivariado de forma determinística ${ }^{22}$. Foram estimados três cenários, com o intuito de testar variaçóes de custos das tecnologias e/ou agravamento dos eventos de sangramento e retrombose.

No primeiro cenário, foi avaliada a redução de 25\% sob o custo da tecnologia de referência. $\mathrm{O}$ segundo e terceiro cenários basearam-se no agravamento do desfecho de sangramento, sendo no segundo cenário estimado um maior grau de sangramento e, no terceiro cenário, uma redução em $50 \%$ do custo da tecnologia de referência somada à utilização de complexo protrombínico. Este estudo não aplicou gráfico de tornado e não realizou análise de sensibilidade probabilística do tipo Monte Carlo.

\section{IMPACTO ORÇAMENTÁRIO}

A avaliação de impacto orçamentário baseou-se em dois cenários, um de referência e outro comparador. Cada cenário foi composto pelas duas tecnologias em análise com diferentes proporçóes de uso dentro do sistema de saúde, representando diferentes condiçôes de mercado. Estimou-se um horizonte temporal para cinco anos. $\mathrm{O}$ cenário referência considerou as tecnologias disponíveis durante o ano de 2017 para o tratamento de TVP e suas respectivas proporçóes de consumo, segundo a análise de prontuários da instituição. No que se refere ao cenário referência, a enoxaparina apresentou market share; ou seja, consumo inicial de $40 \%$, sofrendo redução de consumo 
(10\% por ano até o quarto ano, e no último ano uma reduçáo de 5\%), alcançando $10 \%$ de consumo ao longo dos cinco anos analisados; enquanto a rivaroxabana apresentou consumo inicial de $60 \%$ e atingiu $90 \%$ ao longo dos últimos anos.

Foram empregados como ferramenta para cálculo de impacto orçamentário a Tabela Brasileira de Impacto Orçamentário de Tecnologias da Saúde do Ministério da Saúde, desenvolvida para fármacos, disponível para download no sítio da Rede Brasileira de Tecnologias em Saúde, e o Manual de Impacto Orçamentário ${ }^{23,24}$.

Foi escolhido o método epidemiológico para avaliação do impacto orçamentário, visto que esse método é capaz de estimar de forma mais abrangente os indivíduos que podem se beneficiar do tratamento. Foi aplicada uma taxa de inflaçáo de 5\% ao ano. Desse modo, foi considerada a população brasileira no ano de 2017, com 207.660.929 milhóes de habitantes, segundo o Instituto Brasileiro de Geografia e Estatística (IBGE) $)^{25}$. A população elegível para o estudo foi de $71,4 \%$, sendo excluídos menores de 18 anos; e, a partir dessa população, $20 \%$ foram o equivalente à taxa de prevalência de TVP em câncer.

A análise de sensibilidade se baseou em outros três cenários. No primeiro cenário, foi sugerida a redução de $25 \%$ no valor da enoxaparina; no segundo, foi reduzido o consumo de rivaroxabana para $70 \%$ ano longo dos cinco anos de análise; e, no terceiro cenário, estimaram-se a reduçâo do valor da enoxaparina em $50 \%$ e um alcance de $80 \%$ da taxa de consumo da rivaroxabana até o quinto ano.

Para análise dos resultados, foram utilizados o programa estatístico Prisma ${ }^{\circledR}$ e o Microsoft Excel ${ }^{\circledR}, 2010$. O estudo foi avaliado e aprovado pelo Comitê de Ética em Pesquisa do INCA, sob o número CAAE: 54355416.1.0000.5274.

\section{RESULTADOS}

Cento e cinquenta e três pacientes foram incluídos na análise de custo-efetividade, sendo 95 no braço da rivaroxabana e 58 da enoxaparina. Trinta e nove em uso de rivaroxabana e 26 de enoxaparina foram provenientes do estudo realizado por Leira et al. ${ }^{16}$, o qual avaliou o paciente oncológicos com tumores ginecológicos e TVP.

A média de idade dos pacientes foi de $57( \pm 13,2)$ anos para o grupo da rivaroxabana; enquanto, para o da enoxaparina, foi de $54( \pm 16,5)$ anos. Entre os tipos de tumores analisados, os mais observados foram os de câncer ginecológico, com representatividade de 41,0\% (rivaroxabana), e 44,8\% (enoxaparina), seguido de câncer de mama para a rivaroxabana $(23,2 \%)$ e tecido linfático e hematopoiético para a enoxaparina (17,2\%).

Em relação ao estadiamento da doença de base, a maioria dos pacientes apresentou maior prevalência de

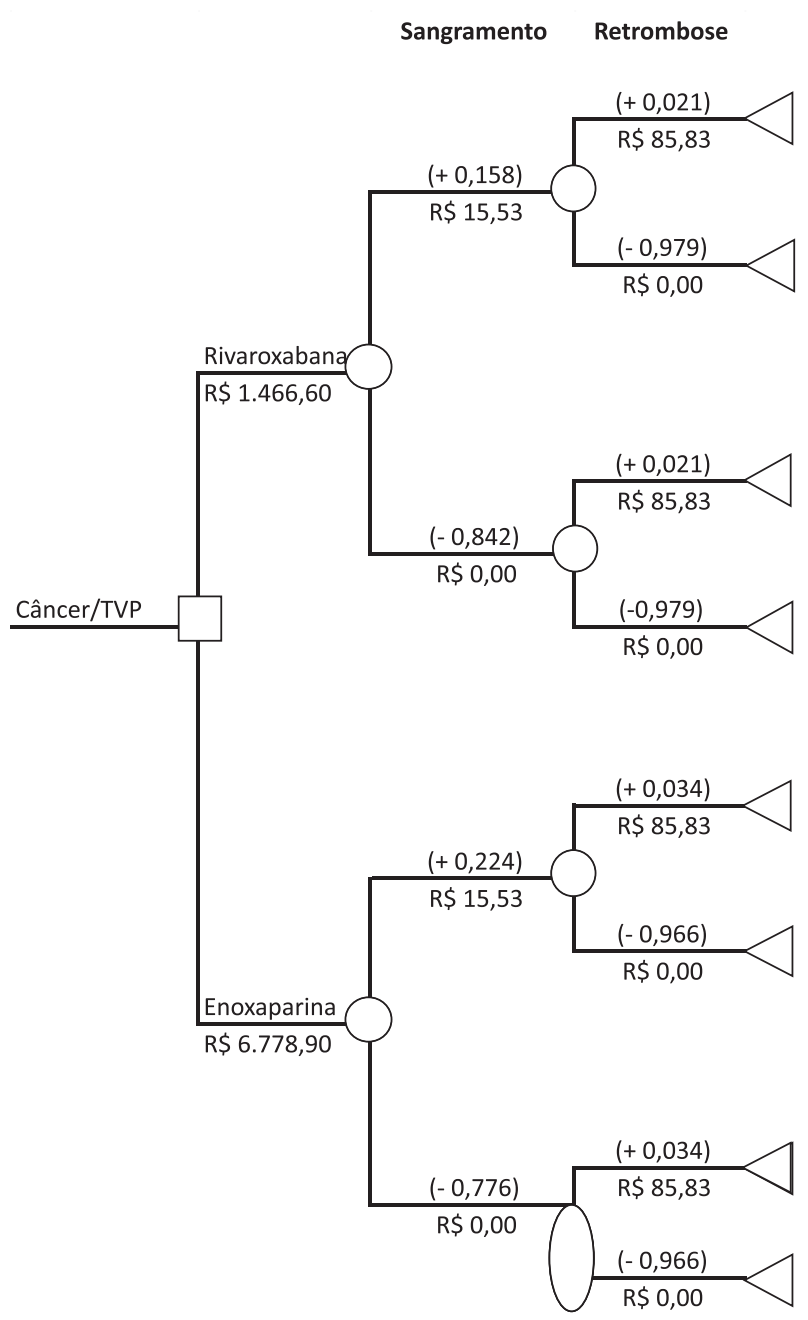

Figura 1. Representação esquemática de árvore de decisão de custo- efetividade da rivaroxabana versus enoxaparina, no tratamento da trombose venosa profunda em pacientes oncológicos

câncer associado à trombose no estadiamento III ou IV, em $55,6 \%$ dos casos. Sendo o grupo da rivaroxabana com $16,8 \%$ e $35,8 \%$ e da enoxaparina com $16,8 \%$ e $39,7 \%$ para os estádios III e IV, respectivamente; $30,7 \%$ dos pacientes não tiveram seu estadiamento localizado em prontuário.

Além disso, foi analisado o tratamento prévio à terapia anticoagulante, no qual foi observado que, dos pacientes que utilizaram a rivaroxabana, $44,2 \%$ foram submetidos à cirurgia, $54,7 \%$ à quimioterapia e $21,1 \%$ à radioterapia prévia. Enquanto, para o braço da enoxaparina, 29,3\% foram submetidos à cirurgia, $70,7 \%$ à quimioterapia e $24,1 \%$ à radioterapia prévia. Houve pacientes que passaram por mais de uma modalidade terapêutica. Contudo, nenhum paciente foi submetido à cirurgia concomitante a anticoagulantes, objetivando reduçáo de risco de hemorragia durante a cirurgia.

Os custos diretos com exame de dopplerfluxometria e consulta médica, bem como custos com os anticoagulantes 
e tratamento para sangramento e retrombose que foram incorporados na árvore de decisão da análise de custo-efetividade, encontram-se representados na Tabela 1.

Em relação aos desfechos, foi observado que 15,8\% dos pacientes apresentaram episódio de sangramento com a utilizaçáo da rivaroxabana, enquanto $22,4 \%$, da enoxaparina. No entanto, essa diferença não foi estatisticamente significativa. Os episódios de retrombose representaram menor frequência quando comparados ao sangramento. Foi identificada retrombose em 2,1\% dos pacientes que utilizaram a rivaroxabana e 3,5\%, a enoxaparina. Ambas as tecnologias apresentaram semelhante efetividade terapêutica conforme descrito na Tabela 1.

A análise de custo-efetividade, por meio da modelo de árvore de decisão (Figura 1), calculou um custo de $\mathrm{R} \$ 1.470,86$ e $\mathrm{R} \$ 6.785,30$ por paciente tratado com rivaroxabana e enoxaparina, respectivamente, por sete meses. A razáo de custo-efetividade incremental observada foi de $\mathrm{R} \$ 5.521,71$ para cada paciente tratado com a enoxaparina ao longo dos sete meses; ou seja, esse valor representa um gasto adicional com o uso da enoxaparina para que o paciente se beneficie com a mesma efetividade anticoagulante. Dessa maneira, a rivaroxabana foi considerada como tecnologia dominante (Tabela 2).

A fim de testar a robustez do modelo de custo-efetividade, foram realizadas análises de sensibilidade (Tabela 3) e propostos três cenários. No primeiro cenário, foi estimada uma redução de $25 \%$ sob o valor do tratamento com a enoxaparina. Já no segundo cenário, foi simulado o agravamento dos desfechos de sangramento, levando em consideração o valor da utilização do ácido
Tabela 2. Razão de custo-efetividade incremental entre rivaroxabana e enoxaparina

\begin{tabular}{lcc}
\hline & $\begin{array}{c}\text { Rivaroxabana } \\
\text { (RS) }\end{array}$ & $\begin{array}{c}\text { Enoxaparina } \\
\text { (RS) }\end{array}$ \\
\hline Custo & $1.470,86$ & $6.785,30$ \\
Custo incremental & - & $5.314,44$ \\
Efetividade & 0,979 & 0,966 \\
Efetividade & - & $-0,013$ \\
incremental & & $7.024,12$ \\
Custo efetividade & $1.502,41$ & $5.521,71$ \\
RCEI & Dominante & \\
\hline
\end{tabular}

Legenda: RCEI = Razão de custo-efetividade incremental.

épsilon aminocaproico, internação, hemogramas, exames, tempo de tromboplastina ativada (TAP) e tempo de protrombina (TTP). E no último cenário, redução em $50 \%$ do valor da enoxaparina e inclusão do uso do tratamento com complexo protrombínico para sangramento maior. Em todos os cenários, a rivaroxabana manteve-se como tecnologia dominante.

Para análise do impacto orçamentário, se fez necessário calcular o valor final mensal de cada tecnologia, o qual considera o valor da tecnologia multiplicado pelas unidades mensais utilizadas para o tratamento, bem como seu custo anual. A rivaroxabana demonstrou valor final mensal de $\mathrm{R} \$ 285,50$, custo anual de $\mathrm{R} \$ 3.426,00$ e adicional anual de $4,4 \%$; enquanto a enoxaparina correspondeu a $\mathrm{R} \$ 1.870,80$ mensais, $\mathrm{R} \$ 22.449,60$ anuais e $0,65 \%$ de adicional anual. $\mathrm{O}$ valor adicional anual representa custos diretos com abordagem, efeitos adversos leves e graves, levando em consideração a frequência anual de cada um, e custos diretos como consulta médica e exames. A

Tabela 1. Parâmetros aplicados à análise de custo-efetividade e impacto orçamentário

\begin{tabular}{lccc} 
& Rivaroxabana & Enoxaparina & p-valor \\
& $\mathbf{n}=95(\%)$ & $\mathbf{n = 5 8 ( \% )}$ & 0,39 \\
Sangramento & 15,79 & 22,41 & 0,63 \\
Retrombose & 2,10 & 3,45 & - \\
Efetividade & $(1-0,021)$ & $(1-0,034)$ & 0,966 \\
Custos unitários (R\$) & 0,979 & & Referência \\
Preço médio dos medicamentos & & 15,80 & BDOMS \\
Dopplerfluxometria & $5,71 / 11,42 *$ & 43,50 & SIGTAP \\
Consulta médica de emergência & 43,50 & 11,00 & SIGTAP \\
Tratamento de sangramento & 11,00 & 15,53 & SIGTAP \\
Tratamento de trombose recorrente & 15,53 & 85,83 & SIGTAP \\
Número de exames & 85,83 & 2 & BDOMS \\
Número de consultas médicas & 2 & 4 & BDOMS \\
Custo do tratamento total (R\$) & 6 & $6.778,90$ & - \\
\hline
\end{tabular}

Legendas: SIGTAP-SUS = Sistema de Gerenciamento da Tabela de Procedimentos, Medicamentos, Órteses, Próteses e Materiais e Medicamentos Especiais do SUS; BDOMS = Base de Dados Oficial do Ministério da Saúde.

Nota *Custo considerado de R 11,42 durante as três primeiras semanas, seguido do custo de R \$ 5,71/dia até o término de sete meses de tratamento. Disponível em: www.comprasgovernamentais.gov.br. 
Tabela 3. Análise de sensibilidade univariada e bivariada determinística da análise de custo-efetividade

\begin{tabular}{|c|c|c|c|c|c|c|}
\hline & \multicolumn{2}{|c|}{ Cenário 1} & \multicolumn{2}{|c|}{ Cenário 2} & \multicolumn{2}{|c|}{ Cenário 3} \\
\hline & $\begin{array}{l}\text { Rivaroxabana } \\
\text { (R\$) }\end{array}$ & $\begin{array}{c}\text { Enoxaparina } \\
\text { (R\$) }\end{array}$ & $\begin{array}{c}\text { Rivaroxabana } \\
\text { (R\$) }\end{array}$ & $\begin{array}{c}\text { Enoxaparina } \\
\text { (R\$) }\end{array}$ & $\begin{array}{l}\text { Rivaroxabana } \\
\text { (RS) }\end{array}$ & $\begin{array}{c}\text { Enoxaparina } \\
\text { (RS) }\end{array}$ \\
\hline Custo & $1.470,86$ & $5.125,59$ & $1.614,92$ & $6.955,23$ & $1.624,12$ & $3.646,71$ \\
\hline $\begin{array}{l}\text { Custo } \\
\text { incremental }\end{array}$ & - & $3.654,73$ & - & $5.340,31$ & - & $2.022,60$ \\
\hline Efetividade & 0,979 & 0,966 & 0,979 & 0,966 & 0,979 & 0,966 \\
\hline $\begin{array}{l}\text { Efetividade } \\
\text { incremental }\end{array}$ & - & $-0,01$ & - & $-0,01$ & - & $-0,01$ \\
\hline $\begin{array}{l}\text { Custo } \\
\text { efetividade }\end{array}$ & $1.502,41$ & $5.305,99$ & $1.649,56$ & $7.200,03$ & $1.658,95$ & $5.754,49$ \\
\hline RCEI & Dominante & - & Dominante & - & Dominante & - \\
\hline
\end{tabular}

Legenda: RCEI = Razão de custo-efetividade incremental.

Notas: Cenário 1: Redução em 25\% no custo da enoxaparina; Cenário 2: Agravamento do desfecho sangramento; e Cenário 3 : Reduçăo em $50 \%$ no custo da enoxaparina mais a utilização do complexo protrombínico.

retrombose foi ponderada como efeito adverso grave e o sangramento como efeito adverso leve. A partir dos dados coletados em prontuários, foi observada frequência de $2 \%$ de efeitos adversos graves com o uso da rivaroxabana e $16 \%$ de efeitos adversos leves. Já para a enoxaparina, foi observada uma frequência de $3 \%$ de efeitos adversos graves e $22 \%$ de leves, dados também obtidos a partir da análise de prontuários. Para o cálculo dos custos, foram adotados os mesmos valores da análise de custo-efetividade.

Foi admitida taxa de inflação de $4,5 \%$ por ano ${ }^{26}$ e horizonte temporal de cinco anos. Em razão da especificidade da utilização dos anticoagulantes, não foram encontradas quotas de mercado baseado em literatura científica, portanto, foi assumida quota para o cenário de referência com base no consumo das tecnologias obtidas por meio de dados coletados para a análise de custo-efetividade, provenientes dos prontuários analisados, nos quais a rivaroxabana teve $60 \%$ de consumo e a enoxaparina $40 \%$. Foi adotado um cenário alternativo e, para esse, foi estimado um alcance de $90 \%$ de consumo até o quinto ano de análise.

A partir da referida análise orçamentária, pode-se visualizar um custo de R\$ 552.994.083.755,33 (Tabela 4), baseado em dados e estimativas do atual panorama de utilização das duas tecnologias anticoagulantes do hospital analisado; enquanto a projeção com o cenário alternativo sugeriu um custo de $\mathrm{R} \$ 467.043 .292 .626,13$ para o gestor ao longo dos cinco anos. Por conseguinte, verificou-se uma potencial economia no impacto orçamentário de $\mathrm{R}$ \$ 85.950.791.129,21 com a possível adoção do cenário alternativo, com crescimento de $60 \%$ para $90 \%$ no consumo da rivaroxabana ao longo dos cinco anos.

Foram adotados três cenários para a análise de sensibilidade (Tabela 4) de impacto orçamentário, sendo esses baseados nas recomendaçôes da Diretriz de Análise de Impacto Orçamentário do Ministério da Saúde ${ }^{23}$.

No primeiro cenário, estimou-se uma reduçáo de $25 \%$ no valor da enoxaparina mantendo o crescimento de $5 \%$ no consumo a cada ano, durante cinco anos e, embora o custo economizado se apresente inferior ao do cenário alternativo estipulado anteriormente, a tecnologia alternativa continuou apontando uma economia, porém reduzida.

Já o segundo cenário de sensibilidade foi estipulado com base em um menor alcance no consumo da tecnologia alternativa. Estimou-se uma porcentagem de consumo de $70 \%$ e, mesmo não havendo alteraçấo dos valores de medicamentos, nesse segundo cenário, a rivaroxabana demonstrou um gasto adicional de $\mathrm{R} \$ 59.710 .659 .587,37$ ao longo dos cinco anos de avaliação.

No terceiro cenário de sensibilidade, com a redução do valor da enoxaparina em $50 \%$ e decréscimo de consumo para $80 \%$ da rivaroxabana, foi observada potencial economia de $\mathrm{R} \$ 4.839 .559 .356,98$ ao longo dos cinco

Tabela 4. Análise de sensibilidade do modelo de impacto orçamentário ao longo de cinco anos pelo método epidemiológico

\begin{tabular}{|lllllll}
\hline \multicolumn{2}{c}{ Cenário 1 } & \multicolumn{2}{c}{ Cenário 2} & \multicolumn{2}{c}{ Cenário 3} \\
\hline Referência (R\$) & Alternativo (RS) & Referência (R\$) & Alternativo (R\$) & Referência (RS) & Alternativo (RS) \\
\hline $441.966 .154 .073,47$ & $381.671 .249 .454,68$ & $552.994 .083 .755,33$ & $612.704 .743 .342,70$ & $331.080 .567 .891,20$ & $326.241 .008 .534,22$ \\
\hline
\end{tabular}

Notas: Cenário 1: Redução em 25\% no valor da enoxaparina; Cenário 2: Redução no consumo da tecnologia alternativa para $70 \%$ ao longo dos cinco anos; Cenário 3: Redução em $50 \%$ do valor da enoxaparina e alcance de $80 \%$ do consumo no mercado da rivaroxabana. 
anos da análise. A fim de verificar o consumo corte para obtenção de economia financeira, foram testadas distintas taxas de consumo da rivaroxabana na Planilha Brasileira de Impacto Orçamentário, e obteve-se um ponto de equilíbrio com o consumo em torno de $79 \%$ da tecnologia em estudo.

Visando a estimar o impacto orçamentário da implantação da rivaroxabana como alternativa para substituiçáo da enoxaparina, entre as medidas clinicamente cabíveis (pacientes com clearence superior a $15 \mathrm{ml} / \mathrm{min}$ e fora do período de quimioterapia), como escolhas para hospitais onde não possuem a rivaroxabana padronizada, foi simulada análise partindo dos mesmos dados, porém, com modificação da taxa de difusáo da tecnologia demandada. A variação aplicada partiu de um cenário referência no qual não há a padronizaçáo da rivaroxabana; ou seja, $0 \%$ de consumo da rivaroxabana e $100 \%$ de uso da enoxaparina, e com aumento gradual anual de $20 \%$ no consumo da tecnologia em estudo até o quinto ano com o alcance de $90 \%$ de consumo dela. Dessa maneira, foi observada a geraçấo de uma economia de $\mathrm{R}$ \$229.442.332.494,48 ao longo de cinco anos pelo cenário proposto.

\section{DISCUSSÃO}

Em um estudo de custo-efetividade realizado no INCA no ano de 2016 com pacientes com cânceres ginecológicos, portadores de TVP, foram observadas frequências de desfechos de segurança (sangramento) e efetividade (retrombose) da rivaroxabana semelhantes aos da enoxaparina. A rivaroxabana, no contexto do estudo, foi apontada como a tecnologia dominante no tratamento ambulatorial da TVP em pacientes com câncer ginecológico ${ }^{16}$. Esses dados corroboram os achados deste estudo, mesmo com a ampliação da população e a diversificaçáo dos casos de cânceres analisados.

Apesar de a rivaroxabana não necessitar de monitoramento laboratorial de rotina, o número médio de exames por pacientes foi semelhante e o número de consultas maior no grupo em uso de rivaroxabana. Essa diferença se justifica pela ainda recente implantação do anticoagulante oral no hospital, além da terapia com DOAC exigir maior monitoramento na fase inicial de tratamento. A baixa média de exames de dopplerfluxometria foi por causa de o seguimento ser indicado quando há alguma expectativa de mudança de conduta ou suspensão da anticoagulação ou suspeita de nova TVP27.

Com relação aos desfechos clínicos, apenas dois pacientes de cada braço apresentaram retrombose. Para esses casos, as intervenções médicas utilizadas foram exames, consulta e conduta medicamentosa. No entanto, na literatura, não há um consenso sobre manejo de retrombose; logo, as condutas que podem ser adotadas são aumento de dose, troca de anticoagulante ou até mesmo inserção de filtro de veia cava em situaçóes especiais ${ }^{28}$.

Entre os pacientes do grupo que foram anticoagulados com a rivaroxabana e demonstraram episódio de sangramento, $23,2 \%$ possuíam como doença de base câncer de mama, porém, diferentemente do esperado, não foram observados pacientes com esse tipo de tumor apresentando episódios de retrombose, levando em consideração que o tratamento hormonal para câncer de mama possui potencial trombogênico. No entanto, o tratamento hormonal, além aumentar o risco de trombose, também pode gerar sangramento ${ }^{29,30}$.

Para pacientes sob a modalidade de tratamento quimioterapia, segundo a padronização da comissão de anticoagulação do hospital, preconiza-se que esses pacientes sejam anticoagulados com a enoxaparina, tendo em vista que os novos anticoagulantes orais, como a rivaroxabana, possuem interaçôes medicamentosas com alguns antineoplásicos, em nível de metabolização, podendo agir como substratos, indutores ou inibidores da CYP3A4, influenciando no tratamento farmacoterapêutico da doença de base ${ }^{31}$. Nesse aspecto, o estudo apontou que $27 \%$ dos pacientes que foram anticoagulados com rivaroxabana receberam o tratamento durante a quimioterapia, o que pode representar um fator de confundimento, tendo em vista que náo é indicado o seu uso em períodos concomitantes com antineoplásicos, por conta das interaçóes farmacocinéticas que podem alterar as concentraçôes plasmáticas do anticoagulante, ocasionando possíveis hemorragias ou retromboses.

Nenhum paciente apresentou sangramento maior; dessa forma, as intervençóes utilizadas em sua maioria foram por meio de exames e medicamentos, sem necessidade de internaçôes. Durante a análise, foram excluídos sangramentos localmente relacionados à doença de base, para reduzir esse tipo de influência, adotando como desfecho de sangramento todos aqueles originados em áreas distintas do tumor, conforme recomendaçóes de Khorana et al. Além disso, é importante ressaltar que, na recomendação para pacientes com tumor gastrintestinal, a farmacoterapia sugerida é a heparina de baixo peso molecular em razão do risco de sangramento ${ }^{15}$.

A razão de custo-efetividade incremental encontrada foi de $\mathrm{R} \$ 5.521,71$, valor este que sofreu pouca influência dos desfechos avaliados, sangramento e retrombose, que, além de baixo custo, foram pouco frequentes. Por consequência, o esquema terapêutico e os custos dos anticoagulantes tiveram influência preponderante na análise final. Somado a isso, a rivaroxabana mostrou-se dominante na análise de sensibilidade, mesmo com o agravamento do desfecho sangramento e a redução dos custos da enoxaparina. Esses dados corroboram os 
encontrados por Leira et al. ${ }^{16}$ e estão alinhados com outros estudos brasileiros ${ }^{17,18}$.

A análise de impacto orçamentário demonstrou que a taxa de consumo da nova tecnologia é o grande norteador do panorama econômico; ou seja, quanto maior a taxa de consumo da rivaroxabana, maior é a economia gerada para o gestor público. A difusão da nova tecnologia com estimativa de aumento de $10 \%$ de consumo até os primeiros quatro anos e $5 \%$ no último ano para a rivaroxabana e concomitante redução proporcional no consumo do tratamento mais custoso, enoxaparina, demonstrou forte contribuição para o panorama econômico, gerando uma economia de $\mathrm{R} \$$ 85.950.791.129,21. Esse resultado reforça o publicado por Piedade et al. ${ }^{18}$, cujo tratamento com a rivaroxabana também demonstrou uma potencial redução de custos quando comparada à enoxaparina/varfarina.

A análise de sensibilidade do impacto orçamentário mostrou-se favorável para a rivaroxabana. A opção pela redução no valor da enoxaparina nos cenários propostos foram realizadas levando em consideração uma competição no mercado farmacêutico, pressupondo uma provável redução no valor da tecnologia comparadora e potencial reposicionamento no mercado.

Pode-se inferir, portanto, que a taxa de difusão da tecnologia do estudo foi a grande influenciadora no impacto orçamentário, uma vez que o terceiro cenário teve o valor da rivaroxabana acrescido em $50 \%$ e taxa de difusão com crescimento em até $80 \%$ e, mesmo assim, demonstrou maior economia com o cenário alternativo.

Do ponto de vista de uma instituição que não possui, como opçáo terapêutica, a rivaroxabana, a inserção do novo anticoagulante oral demonstrou economia em torno de 30\% do impacto orçamentário incremental comparado ao cenário de referência. Dessa maneira, a implantação da nova tecnologia substituindo a enoxaparina em situaçóes clinicamente apropriadas se mostra favorável sob o aspecto econômico, levando em consideração que não houve inferioridade terapêutica e tampouco na segurança do medicamento. Além disso, a forma farmacêutica dos novos anticoagulantes orais pode influenciar positivamente na adesão dos pacientes no tratamento farmacoterapêutico, tendo em vista que a administração da enoxaparina é via subcutânea, podendo acarretar maior desconforto para o paciente durante a administração.

\section{CONCLUSÃO}

A rivaroxabana apresentou náo inferioridade comparada à enoxaparina, com frequência de sangramento e retrombose semelhantes. A razáo de custo-efetividade incremental de $\mathrm{R}$ \$ 5.521,71 para enoxaparina demonstrou uma economia de igual valor para cada paciente tratado com rivaroxabana em sete meses de tratamento.

A análise de impacto orçamentário apontou uma potencial economia de $\mathrm{R} \$ 17.190 .158 .225,84$ por ano. A partir dessa perspectiva, a substituição da enoxaparina pela rivaroxabana, sempre que clinicamente possível, é uma importante estratégia econômica e com potencial benefício para os pacientes e serviços hospitalares.

\section{CONTRIBUIÇÕES}

Todos os autores participaram de todas as etapas do manuscrito e aprovaçáo final da versáo final para publicação.

\section{DECLARAÇÃO DE CONFLITO DE INTERESSES}

Nada a declarar.

\section{FONTES DE FINANCIAMENTO}

Não há.

\section{REFERÊNCIAS}

1. Lyman GH, Khorana AA, Falanga A, et al. American Society of Clinical Oncology guideline: recommendations for venous thromboembolism prophylaxis and treatment in patients with cancer. J Clin Oncol. 2007;25(34):5490505. doi: https://doi.org/10.1200/JCO.2007.14.1283

2. Varki A. Trousseau's syndrome: multiple definitions and multiple mechanisms. Blood. 2007;110(6):1723-9. doi: https://doi.org/10.1182/blood-2006-10-053736

3. Streiff MB, Holmstrom B, Angelini D, et al. NCCN Guidelines Insights: Cancer-Associated Venous Thromboembolic Disease, Version 2.2018. J Natl Compr Canc Netw 2018;16(11):1289-1303. doi: https://doi. org/10.6004/jnccn.2018.0084

4. den Exter PL, Kooiman J, van der Hulle T, et al. New anticoagulants in the treatment of patients with cancerassociated venous thromboembolism. Best Pract Res Clin Haematol. 2013;26(2):163-169.

5. Farge D, Debourdeau P, Beckers M, et al. International clinical practice guidelines for the treatment and prophylaxis of venous thromboembolism in patients with cancer. J Thromb Haemost. 2013 Jan;11(1):56-70. doi: https://doi.org/10.1111/jth.12070

6. Blom JW, Doggen CJ, Osanto S, et al. Malignancies, prothrombotic mutations, and the risk of venous thrombosis. JAMA. 2005;293(6):715-722. doi: https:// doi.org/10.1001/jama.293.6.715

7. Meis E, Levy RA. Câncer e trombose: uma revisão da literatura. Rev Bras Cancerol. 2007;53(2):183-193. 
8. Lyman GH, Bohlke K, Khorana AA, et al. Venous thromboembolism prophylaxis and treatment in patients with cancer: american society of clinical oncology clinical practice guideline update 2014. J Clin Oncol. 2015 Feb 20;33(6):654-6. doi: https://doi.org/10.1200/ JCO.2014.59.7351

9. Mandalá M, Falanga A, Roila F, et al. Management of venous thromboembolism (VTE) in cancer patients: ESMO Clinical Practice Guidelines. Ann Oncol. 2011;22(6 Suppl):vi85-vi92. doi: https://doi. org/10.1093/annonc/mdr392

10. Prins MH, Lensing AW, Brighton TA, et al. Oral rivaroxaban versus enoxaparin with vitamin $\mathrm{k}$ antagonist for the treatment of symptomatic venous thromboembolism in patients with cancer (EINSTEINDVT and EINSTEIN-PE): a pooled subgroup analysis of two randomised controlled trials. Lancet Haematol. 2014;1(1):e37-e46. doi: https://doi.org/10.1016/S23523026(14)70018-3

11. Bach M, Bauersachs R. Spotlight on advances in VTE management: CALLISTO and EINSTEIN CHOICE. Thromb Haemost. 2016;116(Suppl 2):S24-S32. doi: https://doi.org/10.1160/TH16-06-0486

12. Young AM, Marshall A, Thirlwall J, et al. Comparison of an oral factor Xa inhibitor with low molecular weight heparin in patients with cancer with venous thromboembolism: results of a randomized trial (SELECT-D). J Clin Oncol. 2018;36(20):2017-2023. doi: https://doi.org/10.1200/JCO.2018.78.8034

13. Raskob GE, van Es N, Verhamme P, et al. Edoxaban for the treatment of cancer-associated venous thromboembolism. N Engl J Med. 2018;378(7):615-624. doi: https://doi. org/10.1056/NEJMoa1711948

14. EINSTEIN Investigators; Bauersachs R, Berkowitz $\mathrm{SD}$, et al. Oral rivaroxaban for symptomatic venous thromboembolism. N Engl J Med. 2010;363(26):2499510. doi: https://doi.org/10.1056/NEJMoa1007903

15. Khorana AA, Noble S, Lee AYY, et al. Role of direct oral anticoagulants in the treatment of cancer-associated venous thromboembolism: guidance from the SSC of the ISTH. J Thromb Haemost. 2018;16(9):1891-4. doi: https://doi.org/10.1111/jth.14219

16. Leira RR, Costa RS. Análise de custo-efetividade da rivaroxabana versus enoxaparina no tratamento da trombose venosa profunda em pacientes com câncer ginecológico. J Bras Econ Saúde. 2018;10(1):2-8. doi: https://doi.org/10.21115/JBES.v10.n1.p2-8

17. Silva AP, Santoni NB, Schiola A, et al. Custo-efetividade e impacto orçamentário da rivaroxabana na prevenção de eventos tromboembólicos em pacientes pós-artroplastia de quadril e joelho em comparação com dabigatrana, enoxaparina e sem profilaxia sob a perspectiva do Sistema de Saúde Suplementar Brasileiro. J Bras Econ Saúde. 2011;3(3):259-68.
18. Piedade AD, Paladini L, Kashiura D, et al. Análise econômica do tratamento de tromboembolismo venoso com rivaroxabana em comparação com enoxaparina seguida de varfarina sob a perspectiva do Sistema de Saúde Suplementar brasileiro. J Bras Econ Saúde. 2017;9(1):109-21. doi: https://doi.org/10.21115/JBES. v9.n1.p109-21

19. Clexane [Internet]. São Paulo: Sanofi-Aventis Farmacêutica Ltda.; 2000. Bula Paciente. [atualizada 2017 mar. 21; acesso 2017 mar. 21]. Disponível em: http://www.anvisa.gov.br/datavisa/fila_bula/ frmVisualizarBula.asp?pNuTransacao $=4595782017 \& \mathrm{p}$ IdAnexo=5453202

20. Kaatz S, Ahmad D, Spyropoulos AC, et al. Definition of clinically relevant non-major bleeding in studies of anticoagulants in atrial fibrillation and venous thromboembolic disease in non-surgical patients: communication from the SSC of the ISTH. J Thromb Haemost. 2015;13(11):2119-26. doi: https://doi. org/10.1111/jth.13140

21. Bates SM, Jaeschke R, Stevens SM, et al. Diagnosis of DVT: antithrombotic therapy and prevention of thrombosis, 9th ed: American College of Chest Physicians evidence-based clinical practice guidelines. Chest. 2012;141(2 Suppl):e351S-e418S. doi: https:// doi.org/10.1378/chest.11-2299

22. Nita ME, Secoli SR, Nobre MRC, et al. Avaliaçáo de tecnologias em saúde: evidência clínica, análise econômica e análise de decisão. São Paulo: Artmed; 2010.

23. Ministério da Saúde (BR), Secretaria de Ciência, Tecnologia e Insumos Estratégicos, Departamento de Ciência e Tecnologia. Diretrizes metodológicas: análise de impacto orçamentário: manual para o sistema de saúde do Brasil. Brasília: Ministério da Saúde; 2014.

24. Rede Brasileira de Avaliação Tecnologia e Saúde. Planilha brasileira de impacto orçamentário de tecnologias da saúde [Internet]. Brasília, DF: REBRATS; 2011. [acesso 2018 nov. 1]. Disponível em: http://rebrats.saude.gov.br/instrumentoscomplementares?download=114:planilha-brasileira-deimpacto-orcamentario-de-tecnologias-da-saude

25. Instituto Brasileiro de Geografia e Estatística. População residente enviada ao Tribunal de Contas da União - 20012017 [Internet]; 2017 [acesso 2017 fev. 20]. Disponível em: ftp://ftp.ibge.gov.br/Estimativas_de_Populacao/ Estimativas_2017/serie_2001_2017_TCU.pdf

26. Banco Central do Brasil [Internet]. Brasília, DF: Banco Central do Brasil. [data desconhecida]. [acesso $2017 \mathrm{fev}$. 11]. Disponível em: https://www.bcb.gov.br/pt-br/\#!/ home.

27. Renni MJP, Cerqueira MH, Trugilho IA, et al. Mecanismos do tromboembolismo venoso no câncer: uma revisão da literatura. J Vasc Bras. 2017 out.-dez.; 16(4):308-313. doi: http://dx.doi.org/10.1590/16775449.007817 
28. Romualdi E, Ageno W. Management of recurrent venous thromboembolism in cancer patients. Thromb Res. 2016;140(Suppl 1):S128-31. doi: https://doi. org/10.1016/S0049-3848(16)30111-6

29. Mandalá M, Barni S, Prins M, et al. Acquired and inherited risk factors for developing venous thromboembolism in cancer patients receiving adjuvant chemotherapy: a prospective trial. Ann Oncol. 2010;21(4):871-876. doi: https://doi.org/10.1093/annonc/mdp354

30. Onitilo AA, Doi SA, Engel JM, et al. Clustering of venous thrombosis events at the start of tamoxifen therapy in breast cancer: A population-based experience. Thromb Res. 130(1):27-31. doi: https://doi.org/10.1016/j. thromres.2011.11.025

31. Short NJ, Connors JM. New Oral Anticoagulants and the Cancer Patient. Oncologist. 2014;19(1):82-93. doi: https://doi.org/10.1634/theoncologist.2013-0239

Recebido em: 10/6/2019

Aprovado em: 27/8/2019 